\title{
A New Technique of Laplace Variational Iteration Method for Solving Space-Time Fractional Telegraph Equations
}

\author{
Fatima A. Alawad, ${ }^{1}$ Eltayeb A. Yousif, ${ }^{1,2}$ and Arbab I. Arbab ${ }^{3}$ \\ ${ }^{1}$ Department of Mathematics, Faculty of Science, Northern Border University, P.O. Box 1231, Arar 91431, Saudi Arabia \\ ${ }^{2}$ Department of Applied Mathematics, Faculty of Mathematical Sciences, University of Khartoum, P.O. Box 321, 11111 Khartoum, Sudan \\ ${ }^{3}$ Department of Physics, Faculty of Science, University of Khartoum, P.O. Box 321, 11111 Khartoum, Sudan
}

Correspondence should be addressed to Fatima A. Alawad; btal.awad@gmail.com

Received 7 July 2013; Accepted 8 November 2013

Academic Editor: D. D. Ganji

Copyright (C) 2013 Fatima A. Alawad et al. This is an open access article distributed under the Creative Commons Attribution License, which permits unrestricted use, distribution, and reproduction in any medium, provided the original work is properly cited.

In this paper, the exact solutions of space-time fractional telegraph equations are given in terms of Mittage-Leffler functions via a combination of Laplace transform and variational iteration method. New techniques are used to overcome the difficulties arising in identifying the general Lagrange multiplier. As a special case, the obtained solutions reduce to the solutions of standard telegraph equations of the integer orders.

\section{Introduction}

Fractional differential equations are widely used in many branches of sciences. Many phenomena in engineering, physics, chemistry and other sciences can be described successfully using fractional calculus. Nonlinear oscillation of earthquake, acoustics, electromagnetism, electrochemistry, diffusion processes and signal processing can be modeled by fractional equations [1-5]. The telegraph equations have a wide variety of application in physics and engineering. The applications arise, for example, in the propagation of electrical signals and optimization of guided communication systems [6-9]. It is recently shown by Arbab [10] that a quaternionic momentum eigenvalue produces a telegraph equation. This equation is found to describe the propagation of a quantum particle.

The fractional telegraph equations have been investigated by many authors in recent years. Garg et al. [9] derived a solution of space-time fractional telegraph function in a bounded domain by the method of generalized differential transform and obtained the solution in terms of MittageLeffler functions. Chen et al. [11] obtained the solution of nonhomogenous time-fractional telegraph equation with nonhomogenous boundary conditions, namely, Dirichlet, Neumann, and Robin boundary conditions using the method of separation of variables. The solutions are given in the form of the multivariate Mittage-Leffler functions. Ansari [12] derived a formal solution of the time-fractional telegraph equation by applying a fractional exponential operator. Huang [13] considered the time-fractional telegraph equation for the Cauchy problem and signaling problem. He solved the problem by the combined Fourier-Laplace transforms. Also, Huang derived the solution for the bounded problem in a bounded-space domain by means of Sine-Laplace transforms methods. Das et al. [14] used a homotopy analysis method in approximating an analytical solution for the time-fractional telegraph equation and different particular cases have been derived. Jiang and Lin [15] obtained the solution in a series form for the time-fractional telegraph equation with Robin boundary value conditions using the reproducing kernel theorem. Khan et al. [16] used a method based on perturbation theory and Laplace transformation for solving space-time fractional telegraph equations. They considered fractional Taylor series and fractional initial conditions in deriving the solution. Sevimlican [6] considered a one-dimensional space fractional telegraph equations by the variation iteration method; he found the general Lagrange multiplier to be $\lambda=$ $(\xi-x)$. But, as mentioned by $\mathrm{He}[17]$ the exact identification of the general Lagrange multiplier is impossible for most problems and an approximate identification is always followed. He 
[17], approximated the Lagrange multiplier (as $\lambda=-(\xi-x)$ ) for a one-dimensional space-fractional telegraph equations.

Recently, a method that combined the Laplace transform and variational iteration method (LVIM) has been introduced by many authors in solving various types of problems. Abassy et al. [18] used a combination of variational iteration method, Laplace transform, and Pade' technique in obtaining solution to nonlinear equations in compact form. Hammouch and Mekkaoui [19] approximated the solutions of a homogenous Smoluchowski coagulation equation by Laplace variational iteration method. Arife and Yildirim [20] developed Laplace variational iteration method (LVIM) for solving eighth-order equations.

In this paper, the authors extend Laplace variational iteration method (LVIM) and apply it to space-time onedimensional fractional telegraph equations in a half-space domain (signaling problem). This approach enables us to overcome the difficulties that arise in finding the general Lagrange multiplier.

In Section 2, we provide some preliminaries. Section 3 introduces the concept of variational iteration method, while Section 4 illustrates the construction of Laplace variational technique. In Section 5 the authors provide numerical examples. The conclusions are given in Section 6.

\section{Preliminaries}

Definition 1. The Caputo fractional derivative of order $\alpha>0$ of a function $f(x), x>0$ is defined by $[5,21]$

$$
\begin{aligned}
& { }_{0} D_{x}^{\alpha} f(x) \\
& = \begin{cases}\frac{1}{\Gamma(n-\alpha)} \int_{0}^{x}(x-t)^{n-\alpha-1} f^{(n)}(t) d t, & n-1<\alpha \leq n \in \mathbb{N} \\
\frac{d^{n}}{d x^{n}} f(x), & \alpha=n \in \mathbb{N},\end{cases}
\end{aligned}
$$

where ${ }_{0} D_{x}^{\alpha}$ is called the Caputo derivative operator.

Note 1. From Definition 1, the following result is obtained:

$$
\begin{aligned}
& { }_{0} D_{t}^{\alpha} t^{\beta} \\
& \quad= \begin{cases}\frac{\Gamma(\beta+1)}{\Gamma(\beta-\alpha+1)} t^{\beta-\alpha}, & n-1<\alpha \leq n, \beta>n-1, \beta \in \mathscr{R} \\
0, & n-1<\alpha \leq n, \beta \leq n-1 .\end{cases}
\end{aligned}
$$

Definition 2. The Laplace transform of fractional order derivative, is defined by $[1-3,21,22]$

$$
\begin{array}{r}
\mathscr{L}\left[{ }_{0} D_{x}^{\alpha} f(x)\right]=s^{\alpha} \mathscr{L}[f(x)]-\sum_{k=0}^{n-1} s^{\alpha-k-1}\left[f^{(k)}(x)\right]_{x=0} \\
n-1<\alpha \leq n, n \in \mathbb{N} .
\end{array}
$$

Definition 3. The Mittag-Leffler function with two parameters is defined by $[21,23,24]$

$$
\begin{aligned}
& E_{\alpha, \beta}(z) \\
& \quad=\sum_{n=0}^{\infty} \frac{z^{n}}{\Gamma(\alpha n+\beta)}, \quad \alpha, \beta, z \in \mathbb{C}, \operatorname{Re}(\alpha)>0, \operatorname{Re}(\beta)>0 .
\end{aligned}
$$

Note 2. It follows Definition 3 that

$$
\begin{aligned}
& \text { (1) } E_{2,1}\left(x^{2}\right)=\cosh (x), \\
& \text { (2) } E_{2,2}\left(x^{2}\right)=\frac{\sinh (x)}{x}, \\
& \text { (3) } E_{2,3}\left(x^{2}\right)=\frac{1}{x^{2}}[-1+\cosh (x)] .
\end{aligned}
$$

Definition 4. The generalized Mittag-Leffler function is defined by $[23,24]$

$$
\begin{aligned}
& E_{\alpha, \beta}^{\gamma}(z) \\
& \quad=\sum_{k=0}^{\infty} \frac{(\gamma)_{k}}{k ! \Gamma(k \alpha+\beta)}, \quad \alpha, \beta, \gamma \in \mathbb{C}, \operatorname{Re}(\alpha)>0, \operatorname{Re}(\beta)>0,
\end{aligned}
$$

where $(\gamma)_{k}=\gamma(\gamma+1), \ldots,(\gamma+k-1)=\Gamma(\gamma+k) / \Gamma(\gamma)$. For $\gamma=1, E_{\alpha, \beta}^{\gamma}(z)$ reduces to Mittage-Leffler function (4).

\section{Variational Iteration Method}

He [25] developed the variational iteration method (VIM) that is widely used to evaluate either exact or approximate solutions of linear and nonlinear problems [17, 26-28]. The variational iteration method gives the solution in a rapidly infinite convergent series. To illustrate the concept of VIM, we consider the following general nonlinear equation with prescribed auxiliary conditions:

$$
L u(x, t)+N u(x, t)=f(x, t),
$$

where $u$ is the unknown function, $L$ and $N$ are linear and nonlinear operators, respectively, and $f$ is the source term. The correction functional for (7) is given as follows:

$$
\begin{aligned}
& u_{n+1}(x, t) \\
& \quad=u_{n}(x, t)+\int_{0}^{x} \lambda\left[L u_{n}(\xi, t)+N \check{u}_{n}(\xi, t)-f(\xi, t)\right] d \xi,
\end{aligned}
$$

where $\lambda$ is a general Lagrange multiplier that can be identified optimally via the variation theory. The subscript $n$ indicates the $n$th approximation and $\check{u}_{n}$ is considered as a restricted variation $\delta \check{u}_{n}=0$. 


\section{Laplace Variational Iteration Method (LVIM)}

Consider the following general multiterms fractional telegraph equation:

$$
\begin{aligned}
& \frac{\partial^{\alpha} u}{\partial x^{\alpha}}(x, t) \\
& \quad=a_{1} \frac{\partial^{\beta} u}{\partial t^{\beta}}(x, t)+a_{2} \frac{\partial^{\gamma} u}{\partial t^{\gamma}}(x, t)+a_{3} u(x, t)+f(x, t),
\end{aligned}
$$

where $1<\alpha, \beta \leq 2,0<\gamma \leq 1, x, t \geq 0, u(0, t)=h(t)$, $u_{x}(0, t)=g(t)$, and $a_{1}, a_{2}, a_{3}$ are constants.

The new approach of the Laplace variational iteration technique is based on the following steps.

Step 1. Removing the fractional derivative of order $\alpha$ with respect to $x$ for unknown function $u(x, t)$ by using Laplace and inverse Laplace transforms.

Step 2. Differentiating the results obtained in Step 1 with respect to $x$, then we get the value of the general Lagrange multiplier, for the correction functional (iterative formula) to equal one. The concept of the technique is illustrated in the following context.

By applying Laplace transform with respect to $x$, on both sides of (9), we get

$$
\begin{aligned}
& s^{\alpha} \bar{u}(s, t)-s^{\alpha-1} u(0, t)-s^{\alpha-2} u_{x}(0, t) \\
& =\mathscr{L}\left[a_{1} \frac{\partial^{\beta} u(x, t)}{\partial t^{\beta}}+a_{2} \frac{\partial^{\gamma} u(x, t)}{\partial t^{\gamma}}+a_{3} u(x, t)+f(x, t)\right] \\
& \therefore \bar{u}(s, t) \\
& =\frac{1}{s} h(t)+\frac{1}{s^{2}} g(t)+\frac{1}{s^{\alpha}}[\mathscr{L} f(x, t)]+\frac{1}{s^{\alpha}} \mathscr{L} \\
& \quad \times\left[a_{1} \frac{\partial^{\beta} u(x, t)}{\partial t^{\beta}}+a_{2} \frac{\partial^{\gamma} u(x, t)}{\partial t^{\gamma}}+a_{3} u(x, t)\right] .
\end{aligned}
$$

By taking the inverse Laplace transform to (10), we have

$$
\begin{aligned}
& u(x, t) \\
& =h(t)+x g(t)+\mathscr{L}^{-1}\left[\frac{1}{s^{\alpha}} \mathscr{L} f(x, t)\right]+\mathscr{L}^{-1} \\
& \quad \times\left[\frac{1}{s^{\alpha}} \mathscr{L}\left[a_{1} \frac{\partial^{\beta} u(x, t)}{\partial t^{\beta}}+a_{2} \frac{\partial^{\gamma} u(x, t)}{\partial t^{\gamma}}+a_{3} u(x, t)\right]\right] .
\end{aligned}
$$

Now the fractional derivative of order $\alpha$ with respect to $x$ is removed, and the dependent variable $u(x, t)$ in the left hand side of (11), became free of derivatives. Next step, we differentiate (10) with respect to $x$ to get

$$
\begin{aligned}
& \frac{\partial u(x, t)}{\partial x} \\
& =g(t)+\frac{\partial}{\partial x}\left[\mathscr{L}^{-1}\left[\frac{1}{s^{\alpha}} \mathscr{L} f(x, t)\right]\right]+\frac{\partial}{\partial x} \\
& \quad \times\left[\mathscr { L } ^ { - 1 } \left[\frac { 1 } { s ^ { \alpha } } \mathscr { L } \left[a_{1} \frac{\partial^{\beta} u(x, t)}{\partial t^{\beta}}+a_{2} \frac{\partial^{\gamma} u(x, t)}{\partial t^{\gamma}}\right.\right.\right. \\
& \left.\left.\left.+a_{3} u(x, t)\right]\right]\right] .
\end{aligned}
$$

The above step has been taken to enable us to construct the correction functional for (11) to be

$$
\begin{aligned}
u_{n+1} & \\
= & u_{n}(x, t) \\
+ & \int_{0}^{x} \lambda\left\{\frac{\partial u_{n}(\xi, t)}{\partial \xi}-g(t)-\frac{\partial}{\partial \xi}\left[\mathscr{L}^{-1}\left[\frac{1}{s^{\alpha}} \mathscr{L} f(x, t)\right]\right]\right. \\
& -\frac{\partial}{\partial \xi}\left[\mathscr { L } ^ { - 1 } \left[\frac { 1 } { s ^ { \alpha } } \mathscr { L } \left[a_{1} \frac{\partial^{\beta} u_{n}(\xi, t)}{\partial t^{\beta}}\right.\right.\right. \\
& +a_{2} \frac{\partial^{\gamma} u_{n}(\xi, t)}{\partial t^{\gamma}} \\
& \left.\left.\left.\left.+a_{3} u_{n}(\xi, t)\right]\right]\right]\right\} d \xi .
\end{aligned}
$$

The general Lagrange multiplier for (13) can be identified optimally via variation theory to get

$$
1+\left.\lambda\right|_{\xi=x}=0,\left.\quad \grave{\lambda}\right|_{\xi=x}=0 .
$$

From (14), we obtain

$$
\lambda=-1
$$

Substituting $\lambda=-1$ into (13), we get the iterative formula for $n=0,1,2, \ldots$, as follows:

$$
\begin{aligned}
& u_{n+1}= u_{n}(x, t) \\
&-\int_{0}^{x}\left\{\frac{\partial u_{n}(\xi, t)}{\partial \xi}-g(t)-\frac{\partial}{\partial \xi}\left[\mathscr{L}^{-1}\left[\frac{1}{s^{\alpha}} \mathscr{L} f(x, t)\right]\right]\right. \\
&-\frac{\partial}{\partial \xi}\left[\mathscr { L } ^ { - 1 } \left[\frac { 1 } { s ^ { \alpha } } \mathscr { L } \left[a_{1} \frac{\partial^{\beta} u_{n}(\xi, t)}{\partial t^{\beta}}\right.\right.\right. \\
&+a_{2} \frac{\partial^{\gamma} u_{n}(\xi, t)}{\partial t^{\gamma}} \\
&\left.\left.\left.\left.+a_{3} u_{n}(\xi, t)\right]\right]\right]\right\} d \xi .
\end{aligned}
$$


Start with the initial iteration

$$
u_{0}(x, t)=u(0, t)+x u_{x}(0, t)=h(t)+x g(t) .
$$

The exact solution is given as a limit of the successive approximations $u_{n}(x, t), n=0,1,2, \ldots$; in other words, $u(x, t)=\lim _{n \rightarrow \infty} u_{n}(x, t)$.

\section{Numerical Examples}

Example 1. Consider the following space-fractional homogenous telegraph equation:

$$
\begin{aligned}
& \frac{\partial^{\alpha} u(x, t)}{\partial x^{\alpha}} \\
& =\frac{\partial^{2} u(x, t)}{\partial t^{2}}+\frac{\partial u(x, t)}{\partial t}+u(x, t), \quad x, t \geq 0,1<\alpha \leq 2, \\
& u(0, t)=e^{-t}, \quad u_{x}(0, t)=e^{-t} .
\end{aligned}
$$

Solution 1. Applying the Laplace transform with respect to $x$ on both sides of (18), we get

$$
\begin{gathered}
s^{\alpha} \bar{u}(s, t)-s^{\alpha-1} u(0, t)-s^{\alpha-2} u_{x}(0, t) \\
=\mathscr{L}\left[\frac{\partial^{2} u(x, t)}{\partial t^{2}}+\frac{\partial u(x, t)}{\partial t}+u(x, t)\right], \\
\bar{u}(s, t)=\frac{1}{s} e^{-t}+\frac{1}{s^{2}} e^{-t} \\
+\frac{1}{s^{\alpha}} \mathscr{L}\left[\frac{\partial^{2} u(x, t)}{\partial t^{2}}+\frac{\partial u(x, t)}{\partial t}+u(x, t)\right] .
\end{gathered}
$$

The inverse Laplace transform of (19) yields

$$
\begin{aligned}
u(x, t)= & e^{-t}+x e^{-t} \\
& +\mathscr{L}^{-1}\left[\frac{1}{s^{\alpha}} \mathscr{L}\left[\frac{\partial^{2} u(x, t)}{\partial t^{2}}+\frac{\partial u(x, t)}{\partial t}+u(x, t)\right]\right] .
\end{aligned}
$$

Differentiating (20) with respect to $x$, we have

$$
\begin{aligned}
& \frac{\partial u(x, t)}{\partial x} \\
& \quad=e^{-t}+\frac{\partial}{\partial x} \mathscr{L}^{-1}\left[\frac{1}{s^{\alpha}} \mathscr{L}\left[\frac{\partial^{2} u(x, t)}{\partial t^{2}}+\frac{\partial u(x, t)}{\partial t}+u(x, t)\right]\right] .
\end{aligned}
$$

The correction functional for (21) with $\lambda=-1$ is given by

$$
\begin{aligned}
u_{n+1}(x, t) & \\
=u_{n}(x, t)-\int_{0}^{x}[ & \frac{\partial u_{n}(\xi, t)}{\partial \xi}-e^{-t}-\frac{\partial}{\partial \xi} \mathscr{L}^{-1} \\
\times & {\left[\frac { 1 } { s ^ { \alpha } } \mathscr { L } \left[\frac{\partial^{2} u_{n}(\xi, t)}{\partial t^{2}}+\frac{\partial u_{n}(\xi, t)}{\partial t}\right.\right.} \\
& \left.\left.\left.+u_{n}(\xi, t)\right]\right]\right] d \xi .
\end{aligned}
$$

The initial iteration $u_{0}(x, t)=u(0, t)+x u_{x}(0, t)=e^{-t}+x e^{-t}$; then, we have

$$
\begin{aligned}
u_{1}(x, t)= & e^{-t}+x e^{-t}+\frac{x^{\alpha}}{\Gamma(\alpha+1)} e^{-t}+\frac{x^{\alpha+1}}{\Gamma(\alpha+2)} e^{-t} \\
u_{2}(x, t)= & e^{-t}+x e^{-t}+\frac{x^{\alpha}}{\Gamma(\alpha+1)} e^{-t}+\frac{x^{\alpha+1}}{\Gamma(\alpha+2)} e^{-t} \\
& +\frac{x^{2 \alpha}}{\Gamma(2 \alpha+1)} e^{-t}+\frac{x^{2 \alpha+1}}{\Gamma(2 \alpha+2)} e^{-t}, \\
u_{3}(x, t)= & e^{-t}+x e^{-t}+\frac{x^{\alpha}}{\Gamma(\alpha+1)} e^{-t}+\frac{x^{\alpha+1}}{\Gamma(\alpha+2)} e^{-t} \\
& +\frac{x^{2 \alpha}}{\Gamma(2 \alpha+1)} e^{-t}+\frac{x^{2 \alpha+1}}{\Gamma(2 \alpha+2)} e^{-t} \\
& +\frac{x^{3 \alpha}}{\Gamma(3 \alpha+1)} e^{-t}+\frac{x^{3 \alpha+1}}{\Gamma(3 \alpha+2)} e^{-t},
\end{aligned}
$$

Then the general term in successive approximation is given by

$$
u_{n}(x, t)=e^{-t} \sum_{k=0}^{n} x^{k \alpha}\left[\frac{1}{\Gamma(k \alpha+1)}+\frac{x}{\Gamma(k \alpha+2)}\right] .
$$

The solution in a closed form is given by

$$
u(x, t)=\lim _{n \rightarrow \infty} u_{n}(x, t)=e^{-t}\left[E_{\alpha, 1}\left(x^{\alpha}\right)+x E_{\alpha, 2}\left(x^{\alpha}\right)\right]
$$

Letting $\alpha=2$, then

$$
\begin{aligned}
u(x, t) & =e^{-t}\left[E_{2,1}\left(x^{2}\right)+x E_{2,2}\left(x^{2}\right)\right] \\
& =e^{-t}\left[\cosh (x)+x \frac{\sinh (x)}{x}\right]=e^{x-t}
\end{aligned}
$$

The solution is the same as that obtained by Wazwaz [26]. The solution surface of this example is graphically presented in Figure 1 for various fractional orders of $\alpha$.

Example 2. Consider the following space-time fractional nonhomogenous telegraph equation:

$$
\begin{aligned}
\frac{\partial^{\alpha} u(x, t)}{\partial x^{\alpha}}= & \frac{\partial^{2} u(x, t)}{\partial t^{2}}+\frac{\partial^{\gamma} u(x, t)}{\partial t^{\gamma}} \\
& +u(x, t)-x^{2}-t+1, \\
1<\alpha \leq 2, \quad 0<\gamma \leq 1, \quad x, t \geq 0, & 0<0, \quad u_{x}(0, t)=0 .
\end{aligned}
$$




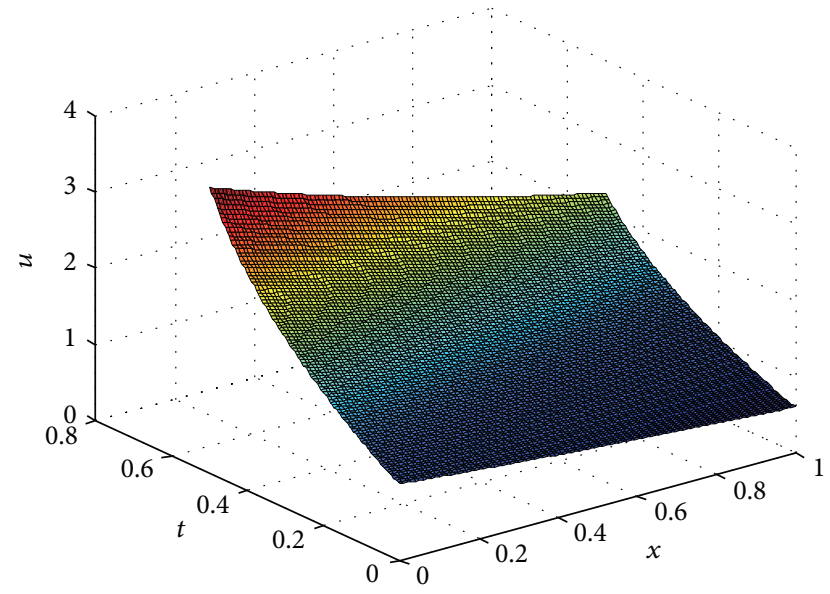

(a)

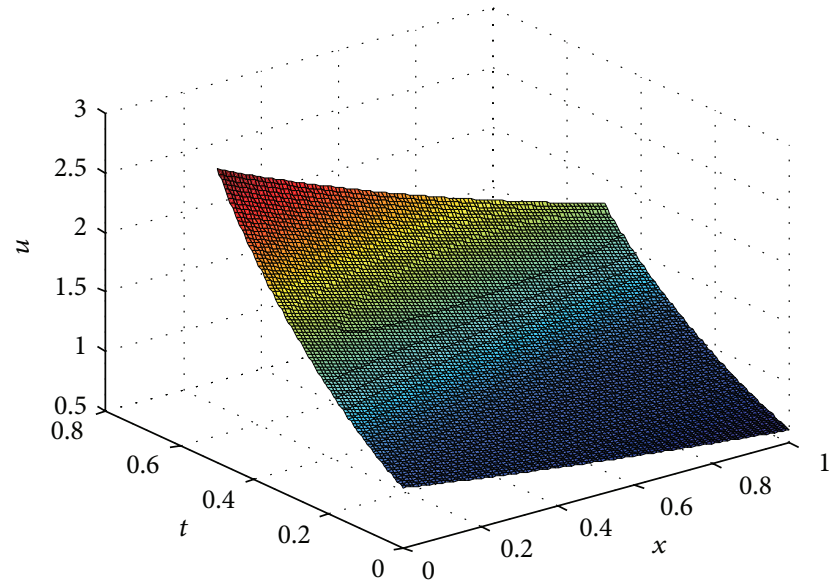

(c)

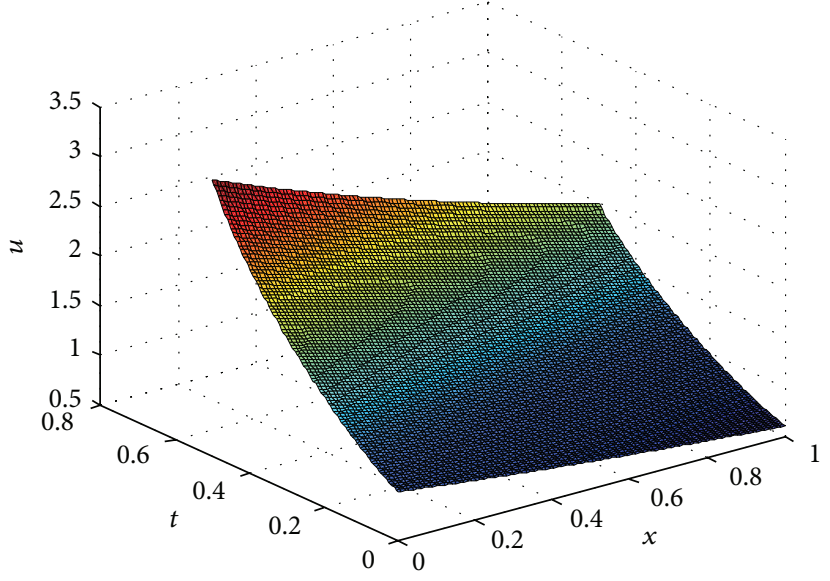

(b)

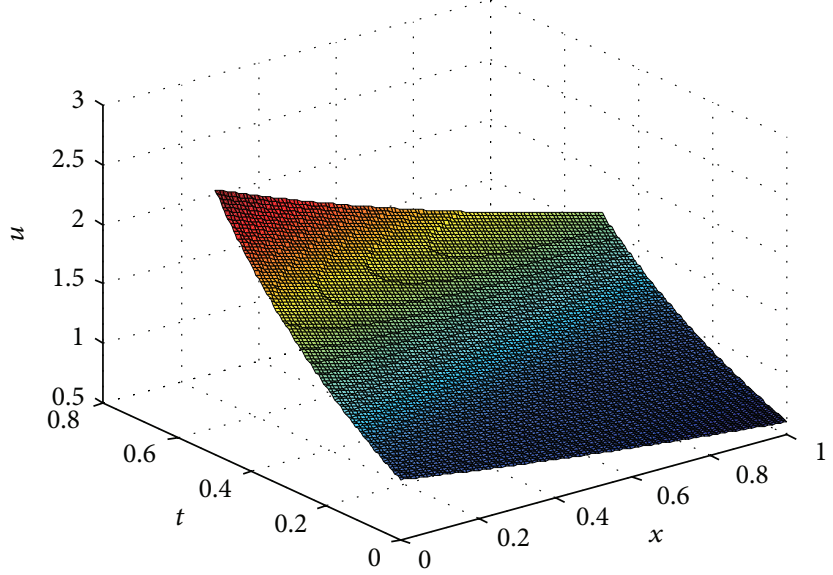

(d)

FIGURE 1: The surface plot of $u(x, t)$ solution of Example 1: (a) $\alpha=1.25$, (b) $\alpha=1.5$, (c) $\alpha=1.75$, and (d) $\alpha=2$.

Solution 2. Taking Laplace transform with respect to $x$ to (27), we get

$$
\begin{aligned}
& s^{\alpha} \bar{u}(s, t)-s^{\alpha-1} u(0, t)-s^{\alpha-2} u_{x}(0, t) \\
& =\mathscr{L}\left[\frac{\partial^{2} u(x, t)}{\partial t^{2}}+\frac{\partial^{\gamma} u(x, t)}{\partial t^{\gamma}}+u(x, t)\right]-\frac{2}{s^{3}}-\frac{t}{s}+\frac{1}{s}, \\
& \bar{u}(s, t) \\
& =\frac{1}{s} t-\frac{2}{s^{\alpha+3}}-\frac{t}{s^{\alpha+1}}+\frac{1}{s^{\alpha+1}} \\
& \quad+\frac{1}{s^{\alpha}} \mathscr{L}\left[\frac{\partial^{2} u(x, t)}{\partial t^{2}}+\frac{\partial^{\gamma} u(x, t)}{\partial t^{\gamma}}+u(x, t)\right] . \\
& \text { The inverse Laplace transform of (28) is given by } \\
& \begin{array}{l}
u(x, t) \\
=
\end{array} \quad \frac{2}{\Gamma(\alpha+3)} x^{\alpha+2}-\frac{t}{\Gamma(\alpha+1)} x^{\alpha}+\frac{1}{\Gamma(\alpha+1)} x^{\alpha} \\
& \quad+\mathscr{L}^{-1}\left[\frac{1}{s^{\alpha}} \mathscr{L}\left[\frac{\partial^{2} u(x, t)}{\partial t^{2}}+\frac{\partial^{\gamma} u(x, t)}{\partial t^{\gamma}}+u(x, t)\right]\right] .
\end{aligned}
$$

Differentiation of (29) with respect to $x$ yields

$$
\begin{aligned}
& \frac{\partial u(x, t)}{\partial x} \\
& =-\frac{2}{\Gamma(\alpha+2)} x^{\alpha+1}-\frac{t}{\Gamma(\alpha)} x^{\alpha-1}+\frac{1}{\Gamma(\alpha)} x^{\alpha-1} \\
& \quad+\frac{\partial}{\partial x}\left[\mathscr{L}^{-1}\left[\frac{1}{s^{\alpha}} \mathscr{L}\left[\frac{\partial^{2} u(x, t)}{\partial t^{2}}+\frac{\partial^{\gamma} u(x, t)}{\partial t^{\gamma}}+u(x, t)\right]\right]\right] .
\end{aligned}
$$

The correction functional for (30) with $\lambda=-1$ is given by

$$
\begin{aligned}
u_{n+1} & \begin{aligned}
u_{n}-\int_{0}^{x}[ & \frac{\partial u_{n}(\xi, t)}{\partial \xi}+\frac{2}{\Gamma(\alpha+2)} \xi^{\alpha+1} \\
& +\frac{t}{\Gamma(\alpha)} \xi^{\alpha-1}-\frac{1}{\Gamma(\alpha)} \xi^{\alpha-1} \\
& -\frac{\partial}{\partial \xi}\left[\mathscr { L } ^ { - 1 } \left[\frac { 1 } { s ^ { \alpha } } \mathscr { L } \left[\frac{\partial^{2} u_{n}(\xi, t)}{\partial t^{2}}\right.\right.\right.
\end{aligned}
\end{aligned}
$$




$$
\begin{aligned}
& +\frac{\partial^{\gamma} u_{n}(\xi, t)}{\partial t^{\gamma}} \\
& \left.\left.\left.\left.+u_{n}(\xi, t)\right]\right]\right]\right] d \xi \\
& u_{0}(x, t)=u(0, t)+x u_{x}(0, t)=t, \\
& u_{1}(x, t)=t-\frac{2 x^{\alpha+2}}{\Gamma(\alpha+3)}+\frac{x^{\alpha}}{\Gamma(\alpha+1)}\left[1+\frac{t^{1-\gamma}}{\Gamma(2-\gamma)}\right] \text {, } \\
& u_{2}(x, t)=t-\frac{2 x^{\alpha+2}}{\Gamma(\alpha+3)}+\frac{x^{\alpha}}{\Gamma(\alpha+1)}\left[1+\frac{t^{1-\gamma}}{\Gamma(2-\gamma)}\right] \\
& -\frac{2 x^{2 \alpha+2}}{\Gamma(2 \alpha+3)}+\frac{x^{2 \alpha}}{\Gamma(2 \alpha+1)}\left[1+\frac{t^{1-\gamma}}{\Gamma(2-\gamma)}\right] \text {, } \\
& u_{3}(x, t)=t-\frac{2 x^{\alpha+2}}{\Gamma(\alpha+3)}+\frac{x^{\alpha}}{\Gamma(\alpha+1)}\left[1+\frac{t^{1-\gamma}}{\Gamma(2-\gamma)}\right] \\
& -\frac{2 x^{2 \alpha+2}}{\Gamma(2 \alpha+3)}+\frac{x^{2 \alpha}}{\Gamma(2 \alpha+1)}\left[1+\frac{t^{1-\gamma}}{\Gamma(2-\gamma)}\right] \\
& -\frac{2 x^{3 \alpha+2}}{\Gamma(3 \alpha+3)}+\frac{x^{3 \alpha}}{\Gamma(3 \alpha+1)}\left[1+\frac{t^{1-\gamma}}{\Gamma(2-\gamma)}\right] \text {, } \\
& \vdots \\
& u_{n}(x, t)=t-2 x^{2} \sum_{k=1}^{n} \frac{\left(x^{\alpha}\right)^{k}}{\Gamma(k \alpha+3)} \\
& +\left[1+\frac{t^{1-\gamma}}{\Gamma(2-\gamma)}\right] \sum_{k=1}^{n} \frac{\left(x^{\alpha}\right)^{k}}{\Gamma(k \alpha+1)} . \\
& u(x, t)=\lim _{n \rightarrow \infty} u_{n}(x, t) \\
& =t+x^{2}-\left[1+\frac{t^{1-\gamma}}{\Gamma(2-\gamma)}\right]-2 x^{2} \sum_{n=0}^{\infty} \frac{\left(x^{\alpha}\right)^{k}}{\Gamma(k \alpha+3)} \\
& +\left[1+\frac{t^{1-\gamma}}{\Gamma(2-\gamma)}\right] \sum_{n=0}^{\infty} \frac{\left(x^{\alpha}\right)^{k}}{\Gamma(k \alpha+1)} \\
& =t+x^{2}\left[1-2 E_{\alpha, 3}\left(x^{\alpha}\right)\right] \\
& +\left[1+\frac{t^{1-\gamma}}{\Gamma(2-\gamma)}\right]\left[E_{\alpha, 1}\left(x^{\alpha}\right)-1\right] \text {. }
\end{aligned}
$$

For $\alpha=2$ and $\gamma=1$, then

$$
u(x, t)=t+x^{2}-2-2 x^{2} E_{2,3}\left(x^{2}\right)+2 E_{2,1}\left(x^{2}\right)=t+x^{2} .
$$

The solution surface of this example is graphically presented in Figure 2 for fixed $\gamma$ and various fractional orders of $\alpha$.
Example 3. Consider the following space-time fractional nonhomogenous telegraph equation:

$$
\begin{gathered}
\frac{\partial^{\alpha} u(x, t)}{\partial x^{\alpha}}=\frac{\partial^{\beta} u(x, t)}{\partial t^{\beta}}+\frac{\partial^{\gamma} u(x, t)}{\partial t^{\gamma}}+u(x, t)-x^{2}-t^{2}-2 t, \\
1<\alpha, \quad \beta \leq 2, \quad \frac{2}{3}<\gamma \leq 1, \quad x, t \geq 0, \\
u(0, t)=t^{2}, \quad u_{x}(0, t)=0, \quad 2<\beta+\gamma \leq 3 .
\end{gathered}
$$

Solution 3. Taking Laplace transform with respect to $x$ to (33), we get

$$
\begin{gathered}
s^{\alpha} \bar{u}(s, t)-s^{\alpha-1} u(0, t)-s^{\alpha-2} u_{x}(0, t) \\
=\mathscr{L}\left[\frac{\partial^{\beta} u(x, t)}{\partial t^{\beta}}+\frac{\partial^{\gamma} u(x, t)}{\partial t^{\gamma}}+u(x, t)\right] \\
\quad-\frac{2}{s^{3}}-\frac{t^{2}}{s}-\frac{2 t}{s}, \\
\bar{u}(s, t) \quad \\
=\frac{1}{s} t^{2}+\frac{1}{s^{\alpha}} \mathscr{L}\left[\frac{\partial^{\beta} u(x, t)}{\partial t^{\beta}}+\frac{\partial^{\gamma} u(x, t)}{\partial t^{\gamma}}+u(x, t)\right] \\
-\frac{2}{s^{\alpha+3}}-\frac{t^{2}}{s^{\alpha+1}}-\frac{2 t}{s^{\alpha+1}} .
\end{gathered}
$$

The inverse Laplace transform of (34) is given by

$$
\begin{aligned}
u(x, t)= & t^{2}-\frac{2 x^{\alpha+2}}{\Gamma(\alpha+3)}-\frac{t^{2} x^{\alpha}}{\Gamma(\alpha+1)}-\frac{2 t x^{\alpha}}{\Gamma(\alpha+1)} \\
& +\mathscr{L}^{-1}\left[\frac{1}{s^{\alpha}} \mathscr{L}\left[\frac{\partial^{\beta} u(x, t)}{\partial t^{\beta}}+\frac{\partial^{\gamma} u(x, t)}{\partial t^{\gamma}}+u(x, t)\right]\right] .
\end{aligned}
$$

Differentiation of (35) with respect to $x$ yields

$$
\begin{aligned}
& \frac{\partial u(x, t)}{\partial x} \\
& =-\frac{2 x^{\alpha+1}}{\Gamma(\alpha+2)}-\frac{t^{2} x^{\alpha-1}}{\Gamma(\alpha)}-\frac{2 t x^{\alpha-1}}{\Gamma(\alpha)} \\
& \quad+\frac{\partial}{\partial x}\left[\mathscr{L}^{-1}\left[\frac{1}{s^{\alpha}} \mathscr{L}\left[\frac{\partial^{\beta} u(x, t)}{\partial t^{\beta}}+\frac{\partial^{\gamma} u(x, t)}{\partial t^{\gamma}}+u(x, t)\right]\right]\right] .
\end{aligned}
$$




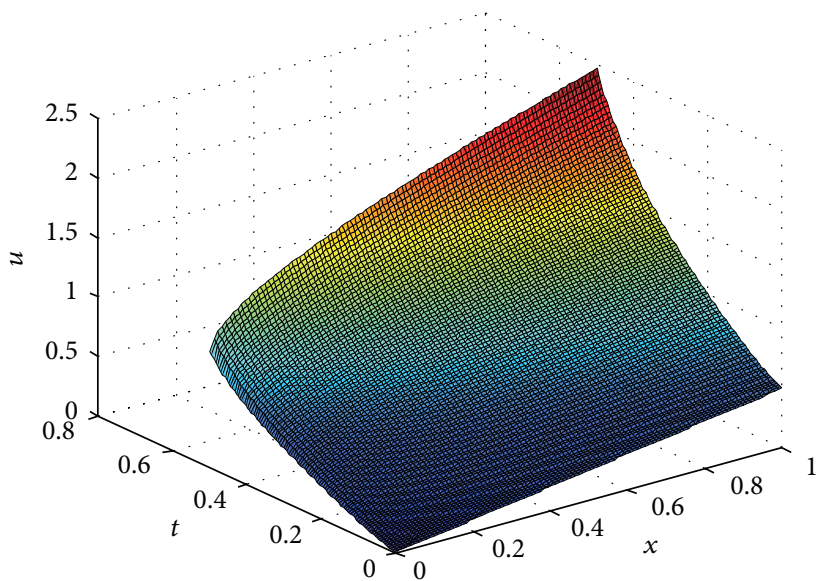

(a)

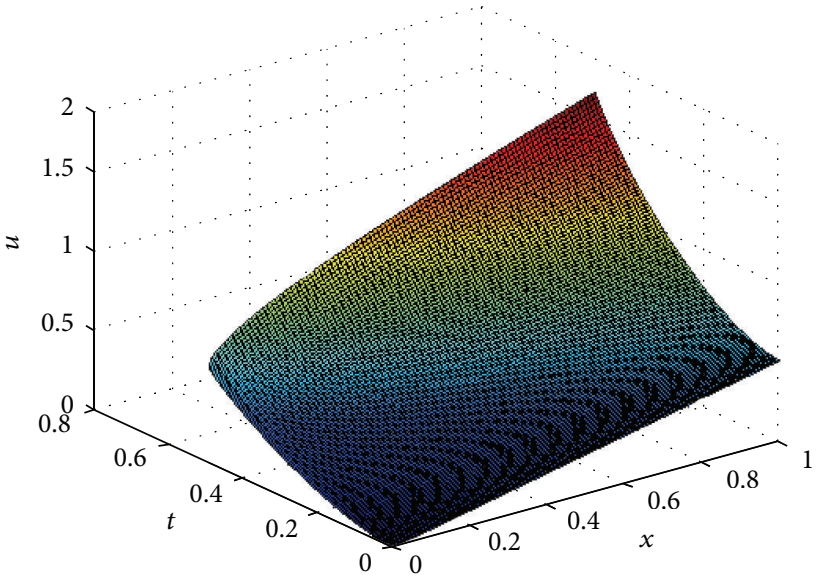

(c)

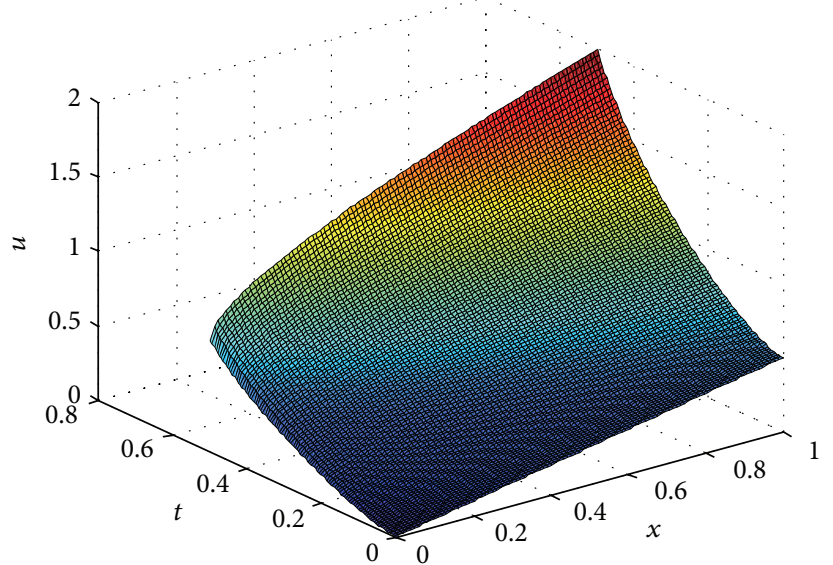

(b)

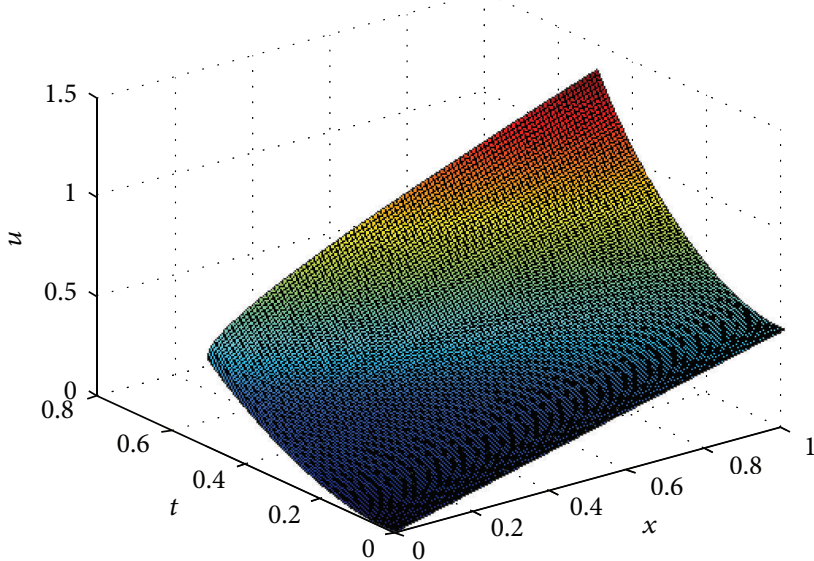

(d)

Figure 2: The surface plot of $u(x, t)$ solution of Example 2 for fixed $\gamma=0.5$ : (a) $\alpha=1.25$, (b) $\alpha=1.5$, (c) $\alpha=1.75$, and (d) $\alpha=2$.

The correction functional for (36) with $\lambda=-1$ is given by

$$
\begin{aligned}
& u_{n+1} \\
& =u_{n}-\int_{0}^{x}\left[\frac{\partial u_{n}(\xi, t)}{\partial \xi}+\frac{2 \xi^{\alpha+1}}{\Gamma(\alpha+2)}+\frac{t^{2} \xi^{\alpha-1}}{\Gamma(\alpha)}+\frac{2 t \xi^{\alpha-1}}{\Gamma(\alpha)}\right. \\
& -\frac{\partial}{\partial \xi}\left[\mathscr { L } ^ { - 1 } \left[\frac { 1 } { s ^ { \alpha } } \mathscr { L } \left[\frac{\partial^{\beta} u_{n}(\xi, t)}{\partial t^{\beta}}\right.\right.\right. \\
& +\frac{\partial^{\gamma} u_{n}(\xi, t)}{\partial t^{\gamma}} \\
& \left.\left.\left.\left.+u_{n}(\xi, t)\right]\right]\right]\right] d \xi \\
& u_{0}(x, t)=u(0, t)+x u_{x}(0, t)=t^{2} \text {, } \\
& u_{1}(x, t)=t^{2}-\frac{2 x^{\alpha+2}}{\Gamma(\alpha+3)} \\
& +\frac{x^{\alpha}}{\Gamma(\alpha+1)}\left[\frac{2 t^{2-\beta}}{\Gamma(3-\beta)}+\frac{2 t^{2-\gamma}}{\Gamma(3-\gamma)}-2 t\right] \text {, } \\
& +\frac{x^{\alpha}}{\Gamma(\alpha+1)}\left[\frac{2 t^{2-\beta}}{\Gamma(3-\beta)}+\frac{2 t^{2-\gamma}}{\Gamma(3-\gamma)}-2 t\right] \\
& -\frac{2 x^{2 \alpha+2}}{\Gamma(2 \alpha+3)}+\frac{x^{2 \alpha}}{\Gamma(2 \alpha+1)} \\
& \times\left[\frac{2 t^{2-2 \gamma}}{\Gamma(3-2 \gamma)}-\frac{2 t^{1-\gamma}}{\Gamma(2-\gamma)}\right. \\
& \left.+\frac{2 t^{2-\beta}}{\Gamma(3-\beta)}+\frac{2 t^{2-\gamma}}{\Gamma(3-\gamma)}-2 t\right], \\
& u_{3}(x, t) \\
& =t^{2}-\frac{2 x^{\alpha+2}}{\Gamma(\alpha+3)} \\
& +\frac{x^{\alpha}}{\Gamma(\alpha+1)}\left[\frac{2 t^{2-\beta}}{\Gamma(3-\beta)}+\frac{2 t^{2-\gamma}}{\Gamma(3-\gamma)}-2 t\right]
\end{aligned}
$$$$
u_{2}(x, t)=t^{2}-\frac{2 x^{\alpha+2}}{\Gamma(\alpha+3)}
$$ 


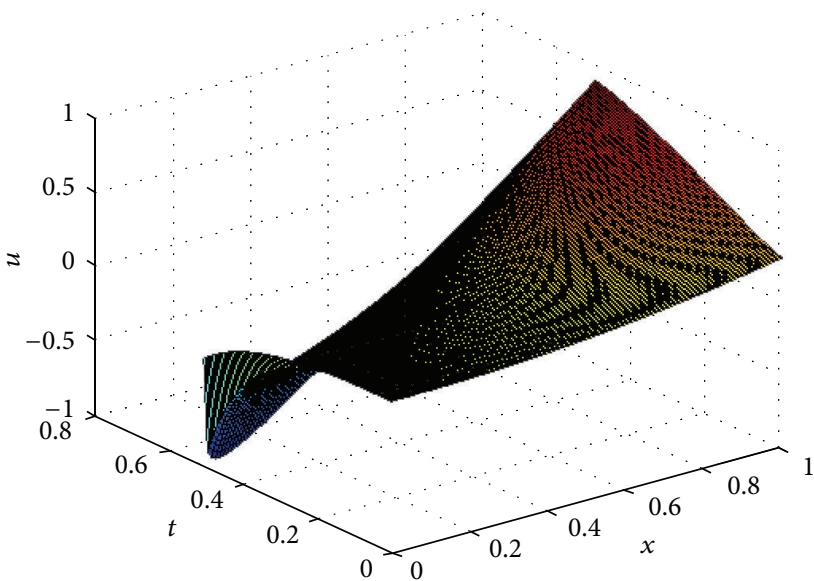

(a)

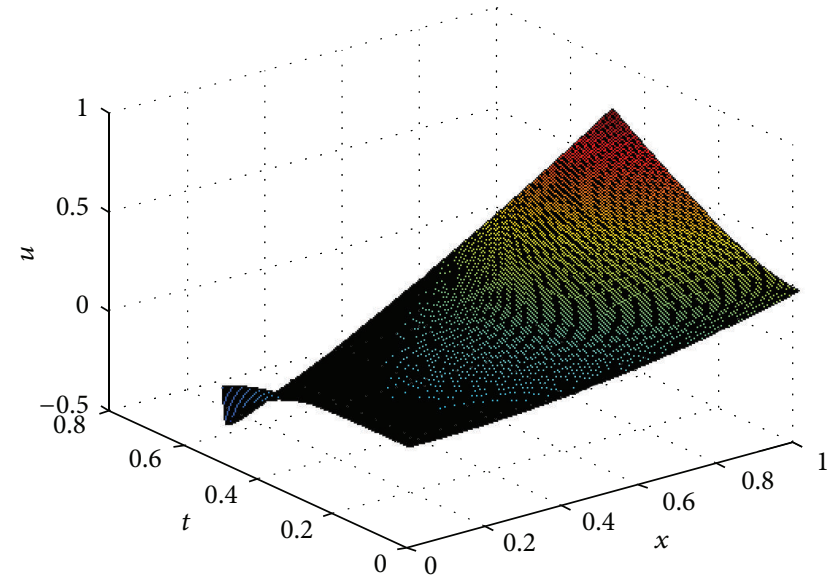

(c)

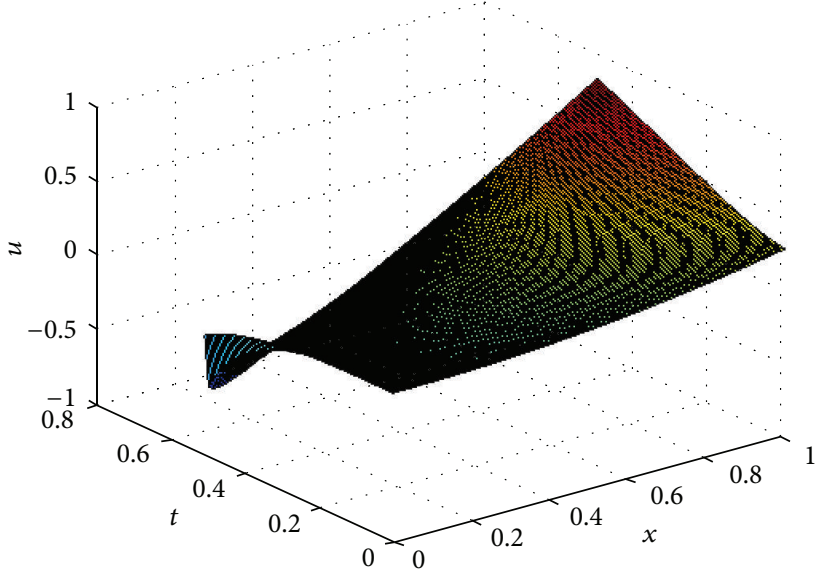

(b)

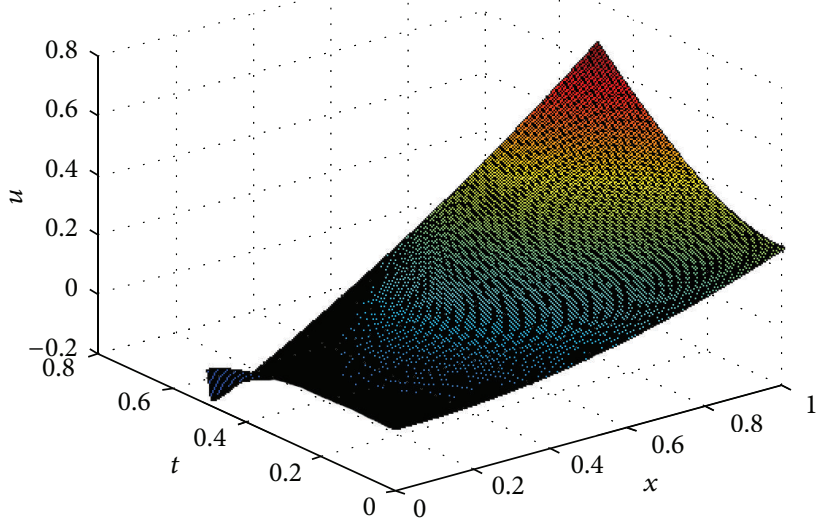

(d)

Figure 3: The surface plot of $u(x, t)$ solution of Example 3 for fixed $\beta=1.25$ and $\gamma=0.75$ : (a) $\alpha=1.25$, (b) $\alpha=1.5$, (c) $\alpha=1.75$, and (d) $\alpha=2$.

$$
\begin{array}{lc}
-\frac{2 x^{2 \alpha+2}}{\Gamma(2 \alpha+3)}+\frac{x^{2 \alpha}}{\Gamma(2 \alpha+1)} & u_{n}(x, t) \\
\times\left[\frac{2 t^{2-2 \gamma}}{\Gamma(3-2 \gamma)}-\frac{2 t^{1-\gamma}}{\Gamma(2-\gamma)}\right. & +\left[\frac{2 t^{2} \sum_{k=1}^{n} \frac{\left(x^{\alpha}\right)^{k}}{\Gamma(k \alpha+3)}}{\Gamma(3-\beta)}+\frac{2 t^{2-\gamma}}{\Gamma(3-\gamma)}-2 t\right] \sum_{k=1}^{n} \frac{\left(x^{\alpha}\right)^{k}}{\Gamma(k \alpha+1)} \\
\left.+\frac{2 t^{2-\beta}}{\Gamma(3-\beta)}+\frac{2 t^{2-\gamma}}{\Gamma(3-\gamma)}-2 t\right] & +\left[\frac{2 t^{2-2 \gamma}}{\Gamma(3-2 \gamma)}-\frac{2 t^{1-\gamma}}{\Gamma(2-\gamma)}\right] \\
-\frac{2 x^{3 \alpha+2}}{\Gamma(3 \alpha+3)}+\frac{x^{3 \alpha}}{\Gamma(3 \alpha+1)} & \times \sum_{k=2}^{n} \frac{(k-1)\left(x^{\alpha}\right)^{k}}{\Gamma(k \alpha+1)}, n \geq 2, \\
\times\left[\frac{4 t^{2-2 \gamma}}{\Gamma(3-2 \gamma)}-\frac{4 t^{1-\gamma}}{\Gamma(2-\gamma)}\right. & u(x, t) \\
\left.+\frac{2 t^{2-\beta}}{\Gamma(3-\beta)}+\frac{2 t^{2-\gamma}}{\Gamma(3-\gamma)}-2 t\right], & =\lim _{n \rightarrow \infty} u_{n}(x, t) \\
\vdots & =t^{2}+x^{2}-\left[\frac{2 t^{2-\beta}}{\Gamma(3-\beta)}+\frac{2 t^{2-\gamma}}{\Gamma(3-\gamma)}-2 t\right]
\end{array}
$$




$$
\begin{aligned}
& -2 x^{2} \sum_{n=0}^{\infty} \frac{\left(x^{\alpha}\right)^{k}}{\Gamma(k \alpha+3)} \\
& +\left[\frac{2 t^{2-\beta}}{\Gamma(3-\beta)}+\frac{2 t^{2-\gamma}}{\Gamma(3-\gamma)}-2 t\right] \sum_{n=0}^{\infty} \frac{\left(x^{\alpha}\right)^{k}}{\Gamma(k \alpha+1)} \\
& +\left[\frac{2 t^{2-2 \gamma}}{\Gamma(3-2 \gamma)}-\frac{2 t^{1-\gamma}}{\Gamma(2-\gamma)}\right] \\
& \times \sum_{k=2}^{\infty} \frac{(k-1)\left(x^{\alpha}\right)^{k}}{\Gamma(k \alpha+1)}, \quad n \geq 2 \\
& =t^{2}+x^{2}\left[1-2 E_{\alpha, 3}\left(x^{\alpha}\right)\right] \\
& +\left[\frac{2 t^{2-\beta}}{\Gamma(3-\beta)}+\frac{2 t^{2-\gamma}}{\Gamma(3-\gamma)}-2 t\right]\left[E_{\alpha, 1}\left(x^{\alpha}\right)-1\right] \\
& +\left[\frac{2 t^{2-2 \gamma}}{\Gamma(3-2 \gamma)}-\frac{2 t^{1-\gamma}}{\Gamma(2-\gamma)}\right] \\
& \times\left[1+E_{\alpha, 1}^{2}\left(x^{\alpha}\right)-2 E_{\alpha, 1}\left(x^{\alpha}\right)\right] .
\end{aligned}
$$

For $\alpha=\beta=2$ and $\gamma=1$, we get the standard equation with the solution

$$
\begin{aligned}
u(x, t) & =t^{2}+x^{2}-2-2 x^{2} E_{2,3}\left(x^{2}\right)+2 E_{2,1}\left(x^{2}\right)+0 \\
& =t^{2}+x^{2} .
\end{aligned}
$$

The solution surface of this example is graphically presented in Figure 3 for fixed $\gamma$ and $\beta$ and various fractional orders of $\alpha$.

\section{Conclusion}

In this paper, a combined form of Laplace transform and variational iteration method is presented to handle spacetime fractional telegraph equations in a half-space domain. The space and time derivatives are considered in the Caputo sense. Certain techniques are used to overcome the complexity of identifying the general Lagrange multiplier. The solutions are obtained in series form that rapidly converges in a closed exact formula with simply computable terms. The calculations are simple and straightforward. The method was tested on three examples on different situations. The technique is powerful, reliable, and efficient. This technique can be extended to solve various linear and nonlinear fractional problems in applied science.

\section{References}

[1] J. Sabatier, O. P. Agrawal, and J. A. T. Machado, Advances in Fractional Calculus: Theoretical Developments and Applications in Physics and Engineering, Springer, New York, NY, USA, 2007.

[2] L. Debnath, "Recent applications of fractional calculus to science and engineering," International Journal of Mathematics and Mathematical Sciences, no. 54, pp. 3413-3442, 2003.
[3] M. Bhatti, "Fractional Schrödinger wave equation and fractional uncertainty principle," International Journal of Contemporary Mathematical Sciences, vol. 2, no. 19, pp. 943-950, 2007.

[4] Z. Z. Ganji, D. D. Ganji, and Y. Rostamiyan, "Solitary wave solutions for a time-fraction generalized Hirota-Satsuma coupled $\mathrm{KdV}$ equation by an analytical technique," Applied Mathematical Modelling, vol. 33, no. 7, pp. 3107-3113, 2009.

[5] R. E. Gutirrez, J. M. Rosario, and J. T. Machado, "Fractional order calculus: basic concepts and engineering applications," Mathematical Problems in Engineering, vol. 2010, Article ID 375858, 19 pages, 2010.

[6] A. Sevimlican, "An approximation to solution of space and time fractional telegraph equations by He's variational iteration method," Mathematical Problems in Engineering, vol. 2010, Article ID 290631, 10 pages, 2010.

[7] D. Campos and V. Méndez, "Different microscopic interpretations of the reaction-telegrapher equation," Journal of Physics A, vol. 42, no. 7, Article ID 075003, 13 pages, 2009.

[8] M. Garg and A. Sharma, "Solution of space-time fractional telegraph equation by Adomian decomposition method," Journal of Inequalities and Special Functions, vol. 2, no. 1, pp. 1-7, 2011.

[9] M. Garg, P. Manohar, and S. L. Kalla, "Generalized differential transform method to space-time fractional telegraph equation," International Journal of Differential Equations, vol. 2011, Article ID 548982, 9 pages, 2011.

[10] A. I. Arbab, "Derivation of Dirac, Klein-Gordon, Schrodinger, diffusion and quantum heat transport equations from the universal wave equation," Europhysics Letters, vol. 92, no. 4, Article ID 40001, 2010.

[11] J. Chen, F. Liu, and V. Anh, "Analytical solution for the timefractional telegraph equation by the method of separating variables," Journal of Mathematical Analysis and Applications, vol. 338, no. 2, pp. 1364-1377, 2008.

[12] A. Ansari, "Fractional exponential operators and timefractional telegraph equation," Boundary Value Problems, vol. 2012, article 125, 2012.

[13] F. Huang, "Analytical solution for the time-fractional telegraph equation," Journal of Applied Mathematics, vol. 2009, Article ID 890158, 9 pages, 2009.

[14] S. Das, K. Vishal, P. K. Gupta, and A. Yildirim, "An approximate analytical solution of time-fractional telegraph equation," Applied Mathematics and Computation, vol. 217, no. 18, pp. 7405-7411, 2011.

[15] W. Jiang and Y. Lin, "Representation of exact solution for the time-fractional telegraph equation in the reproducing kernel space," Communications in Nonlinear Science and Numerical Simulation, vol. 16, no. 9, pp. 3639-3645, 2011.

[16] Y. Khan, J. Diblik, N. Faraz, and Z. Smarda, "An efficient new perturbation Laplace method for space-time fractional telegraph equations," Advance in Difference Equations, vol. 2012, article 204, 2012.

[17] J. H. He, "An aproximation to solution of space and time fractional telegraph equations by the variational iteration method," Mathematical Problems in Engineering, vol. 2012, Article ID 394212, 2 pages, 2012.

[18] T. A. Abassy, M. A. El-Tawil, and H. El-Zoheiry, "Exact solutions of some nonlinear partial differential equations using the variational iteration method linked with Laplace transforms and the Padé technique," Computers \& Mathematics with Applications, vol. 54, no. 7-8, pp. 940-954, 2007. 
[19] Z. Hammouch and T. Mekkaoui, "A Laplace-variational iteration method for solving the homogeneous Smoluchowski coagulation equation," Applied Mathematical Sciences, vol. 6, no. 18, pp. 879-886, 2012.

[20] A. S. Arife and A. Yildirim, "New modified variational iteration transform method (MVITM) for solving eighth-order boundary value problems in one step," World Applied Sciences Journal, vol. 13, no. 10, pp. 2186-2190, 2011.

[21] I. Podlubny, Fractional Differential Equations, vol. 198 of Mathematics in Science and Engineering, Academic Press, New York, NY, USA, 1999.

[22] L. Debnath and D. Bhatta, Integral Transforms and Their Applications, Taylor \& Francis Group,LLC, 2nd edition, 2007.

[23] H. J. Haubold, A. M. Mathai, and R. K. Saxena, "MittagLeffler functions and their applications," Journal of Applied Mathematics, vol. 2011, Article ID 298628, 51 pages, 2011.

[24] M. N. Berberan-Santos, "Properties of the Mittag-Leffler relaxation function," Journal of Mathematical Chemistry, vol. 38, no. 4, pp. 629-635, 2005.

[25] J. H. He, "Variational iteration method-a kind of nonlinear analytical technique: some examples," International Journal of Nonlinear Mechanics, vol. 34, no. 4, pp. 699-708, 1999.

[26] A. M. Wazwaz, Partial Differential Equations and Solitary Waves Theory, Nonlinear Physical Science, Higher Education Press, Beijing, China; Springer, Heidelberg, Germany, 2009.

[27] J. H. He and X. H. Wu, "Variational iteration method: new development and applications," Computers \& Mathematics with Applications, vol. 54, no. 7-8, pp. 881-894, 2007.

[28] S. A. Khuri and A. Sayfy, "A Laplace variational iteration strategy for the solution of differential equations," Applied Mathematics Letters, vol. 25, no. 12, pp. 2298-2305, 2012. 


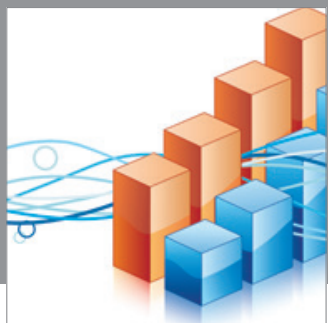

Advances in

Operations Research

mansans

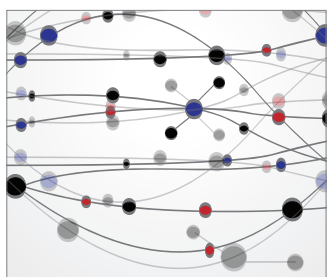

The Scientific World Journal
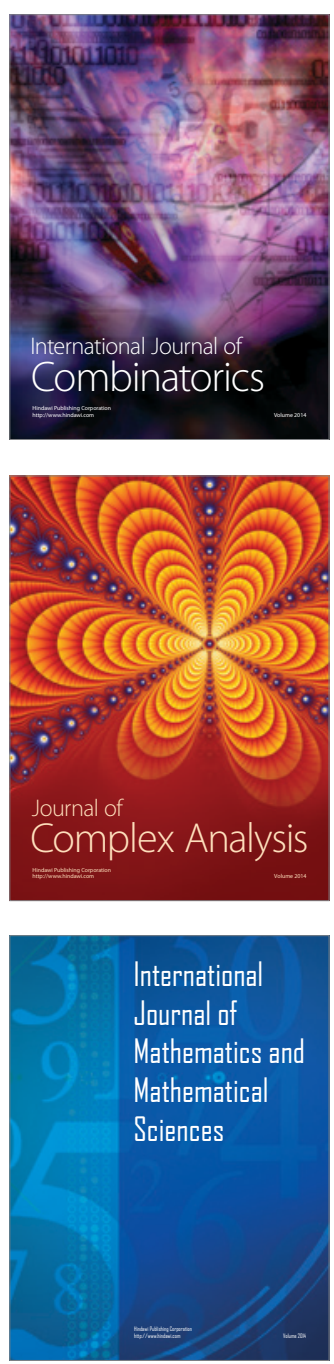
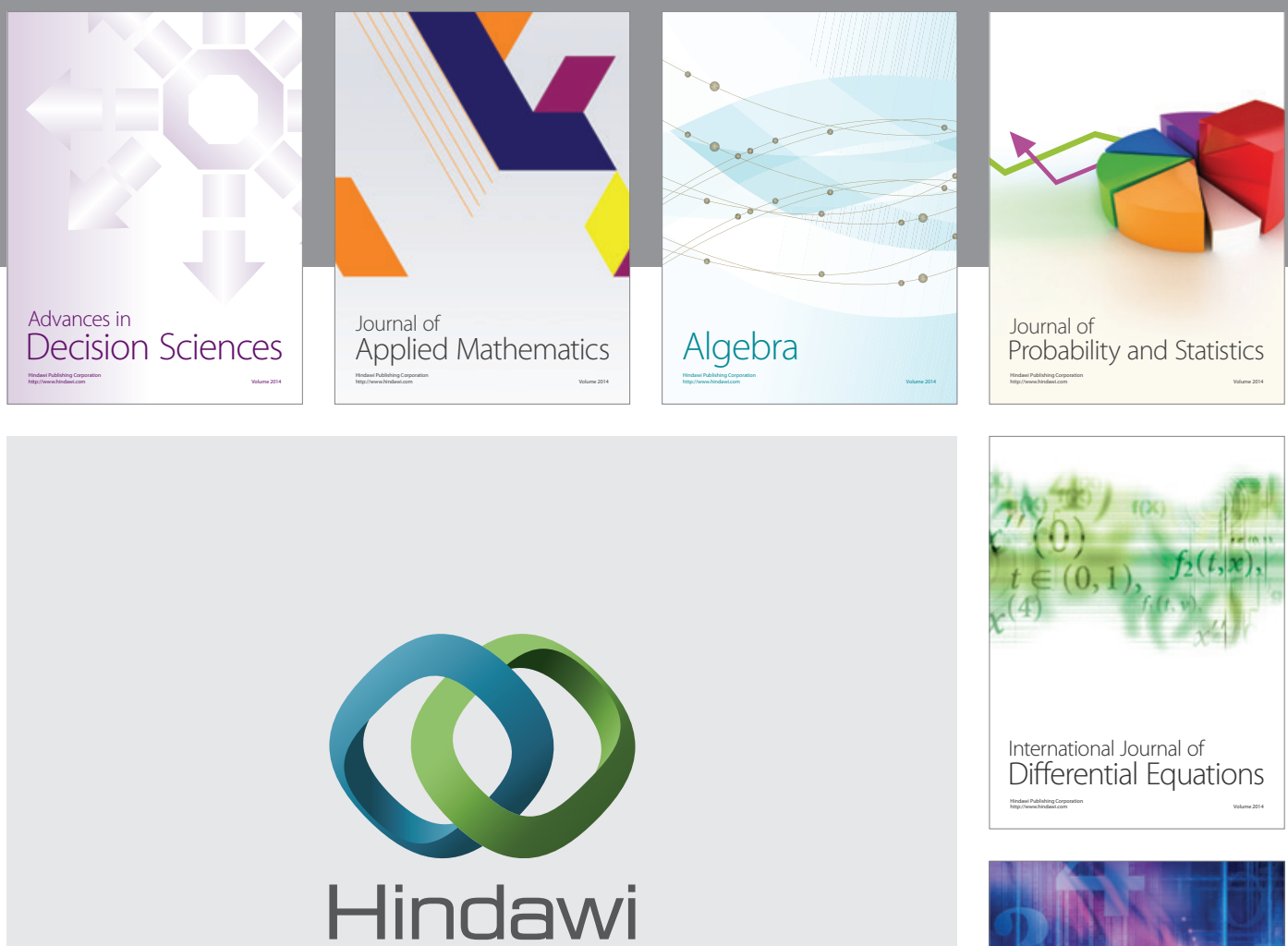

Submit your manuscripts at http://www.hindawi.com
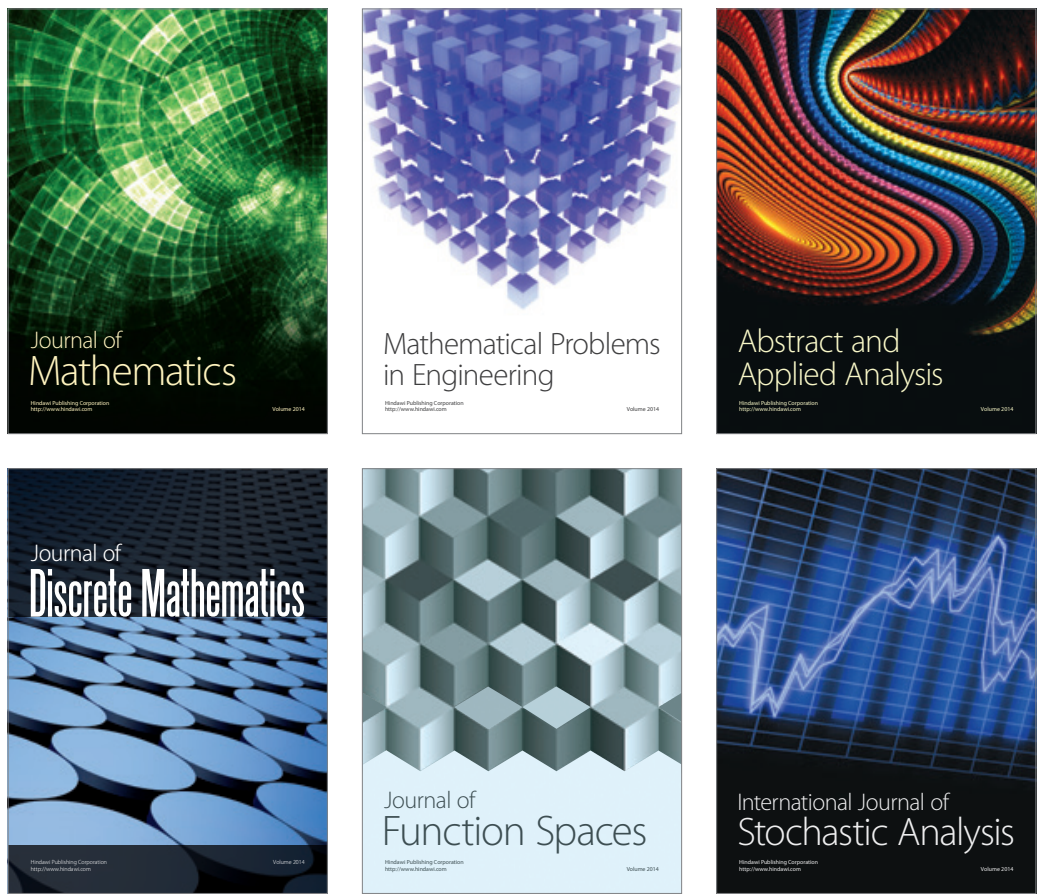

Journal of

Function Spaces

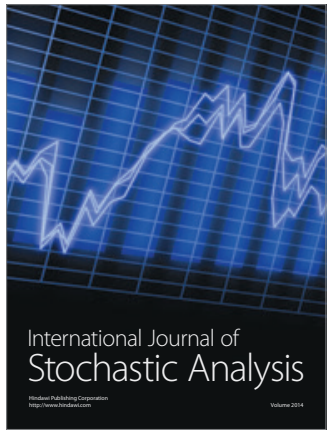

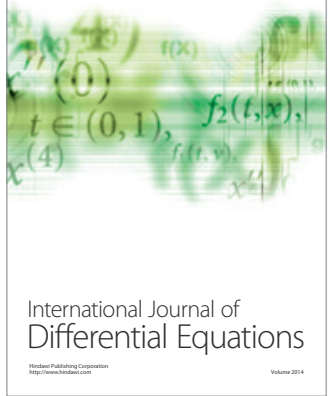
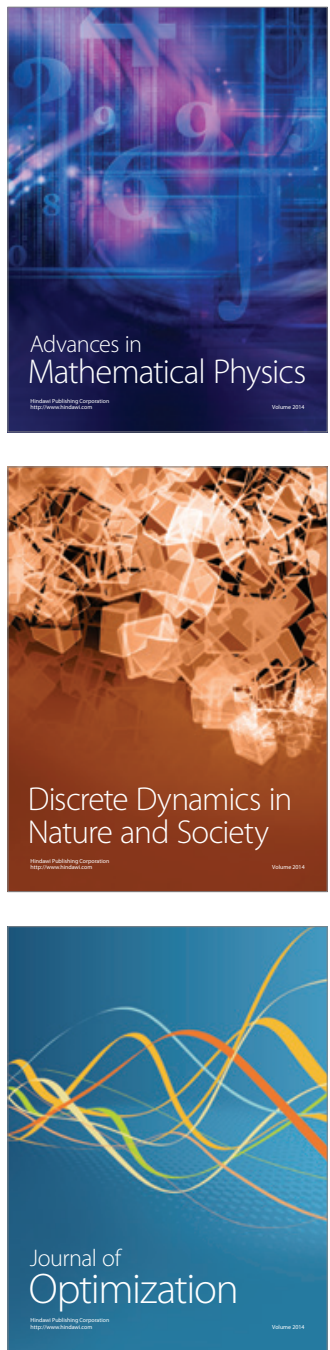